\title{
Stochastic regimes in very-low-frequency fluidic oscillator
}

\author{
Václav Tesaŕ ${ }^{1, a}$ \\ ${ }^{1}$ Institute of Thermomechanics v.v.i., Academy of Sciences of the Czech Republic, Dolejškova 5, 18200 Praha-Kobylisy, Czech Republic
}

\begin{abstract}
Paper discusses interesting unexpected stochastic regimes discovered in a fluidic oscillator designed for operation at very low oscillation frequencies - without the inconvenience of the long feedback loops needed in standard low-frequency oscillator designs. The new oscillator contains a pair of bistable turn-down active valves operating in anti-parallel - essentially analogous to Abraham \& Bloch electric "multibrateur" invented in 1919. Three different self-excited oscillation regimes were found. In the order of increasing supplied flow rate, these regimes are characterised by: (A) generation of stochastic-duration multi-pulse packs, (B) generation of individual pulses with a degree of periodicity, and (C) regime with randomly appearing flow pulses separated by intervals of the order of seconds.
\end{abstract}

\section{Introduction}

This paper describes an unusual oscillator supplied with steady air flow and generating flow pulses in its output terminal. Unusual property of this oscillator is an extremely low frequency - and significant pulse randomness. Laboratory model of the oscillator was investigated for potential use in producing very small, sub-millimetre size gas microbubbles in water. There is a wide range of engineering applications in which the microbubbles bring significant advantages over standard larger size bubbles. It may suffice to name just the use in supplying $\mathrm{CO}_{2}$ to algae cultivated in a bioreactor for producing biofuel [1]. It is an important process, pursued with the ultimate target of reducing the present-day automobile fuel dependence on fossil oil imported from politically unstable regions. The $\mathrm{CO}_{2}$ from the microbubbles is needed for algal photosynthesis and an additional task of $\mathrm{O}_{2}$ removal. The small size increases the area of total collective gas/liquid interface and thus increases the rate of diffusion transport. The fact that nomoving-part fluidic oscillators can generate smaller than usual bubbles by pulsating the supplied gas flow was demonstrated already $\sim 10$ years ago [2], but for a considerable time the details of the microbubble generation mechanism remained unexplained.

Fluidic oscillators, possessing no movable or deformable components, are particularly attractive for the role of the gas flow pulsation in microbubble generation because of their reliability, no need of maintenance, and low cost (since they are nothing more than just a specially shaped gas inlet cavities). Until recently, development of oscillators for this purpose was characterised by demand of very high oscillation frequency. This seemed quite logical because the microbubbles, due to their small size and dominance of relatively strong surface tension forces, posses very high natural frequency of surface oscillation, of the order of kilohertz [3]. It was believed that the small size of the microbubbles is the result of pulsation-promoted fragmentation of initially larger

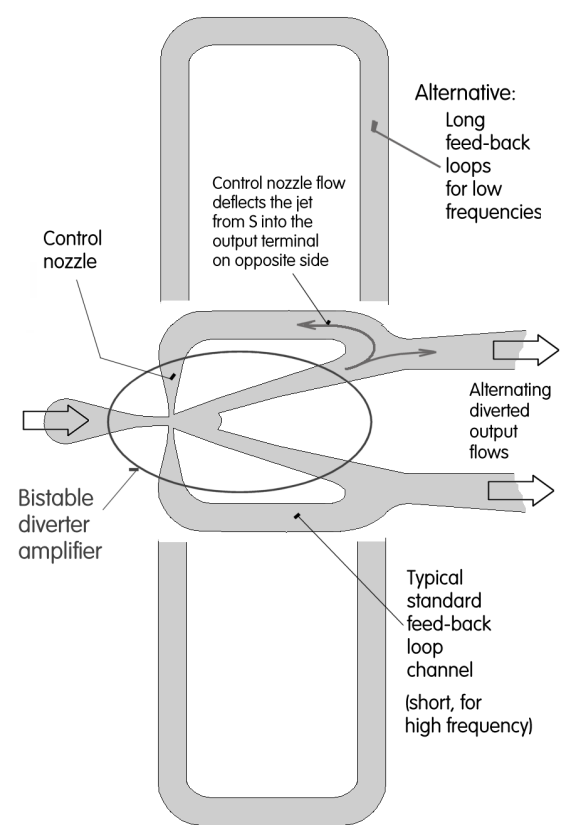

Figure 1. Standard configurations of present-day fluidic oscillators $[6,7]$. The main component is a bistable diverter amplifier. It is provided with feedback loop channels, connecting amplifier outputs with control nozzles. Low oscillation frequency would need very long feedback loops.

bubbles — with the oscillator obviously operating in resonance with this natural frequency.

\footnotetext{
${ }^{\text {a } C o r r e s p o n d i n g ~ a u t h o r: ~ t e s a r @ i t . c a s . c z ~}$
} 
The problem was difficulty in obtaining the high frequency with typical fluidic oscillators operating very much below the required kilohertz range. The present-day oscillators, as shown in Fig. 1, consist of two parts: a) a fluidic amplifier, usually of the bistable jet-diverting type, and b) feedback loops. Typical achievable high frequencies with the version resembling Fig. 1 are near to $f \sim 300 \mathrm{~Hz}$. Somewhat higher frequencies, perhaps up to $f \sim 500 \mathrm{~Hz}$, may be obtained with the recently introduced configuration described in [4], with the loop feedback replaced by signal returned from a resonator. Of course, both frequency values mentioned above are very much below the requested kilohertz regime. A very small oscillator size was necessary, calling for special fabrication techniques and limiting the production rate of the microbubbles. Solutions were sought in special highfrequency versions, e.g., designs facilitating generation of higher harmonics [5].

Quite recently, the target of the development was completely reversed. Performed detailed studies of microbubble formation, using very high speed camera and special objective lenses with very long working distances, have revealed that the key problem is the growth of slowly moving microbubbles at the aerator exit by repeated multiple conjunctions - discussed in Part I of [8]. In the next Part II of [8] it was demonstrated that the conjunctions may be suppressed by reversing, for a part of the oscillation period, the direction of motion in the aerator passages. The microbuble protected inside the passage is thus prevented from contact with other, previously generated microbubbles. It was also found that this working mode, with reversing flow direction of water columns in aerator channels, actually necessitates low driving frequency.

The classical feedback oscillator configuration of Figure 1 is unsuitable for pursuit of the newly emerged target of very low frequencies. It would be necessary to have the feedback loop channels of lengths of the order of metres or even tens of metres [6]. This would make the oscillator loops difficult to stow in the available spaces near the aerator. Also the length - as well as the multiple channel bends, with flow direction changes made in an effort towards compactness — would lead to large hydraulic losses inside the loop. The losses may cause the oscillator to cease operating.

Absence of any success in the attempts to adapt the configuration from Figure 1 to very low frequency regimes has led to recognition of the need to develop new oscillators, based on completely different operation principles.

An inspiration was found in early history of electronics. Design of fluidic circuits has, quite naturally, followed the pattern of electronic circuits as its historical predecessor. Of course, there is a large difference in the final steps of actual realisations. The conceptual similarity of the de-stabilising feedback on one hand and yet different realisation of the idea on the other hand may be seen in the example of the feedback presented in Fig. 1.

\section{Electronic circuits with anti-parallel, mutually blocked valves}

If a designer of an electronic circuit is requested to generate a signal output in the form of a train of rectangular pulses, he has as an option the use of an astable flip-flop circuit. This consists of a pair of electric current-controlling devices operated in anti-parallel, with mutual blockage. This is set up by interconnecting the output terminals of one device to the input terminal of the other one - and vice versa. The result is a circuit switching between two regimes. In one regime the OPEN state in one device is simultaneous with CLOSED state in the other one. In the next regime their role are exchanged.

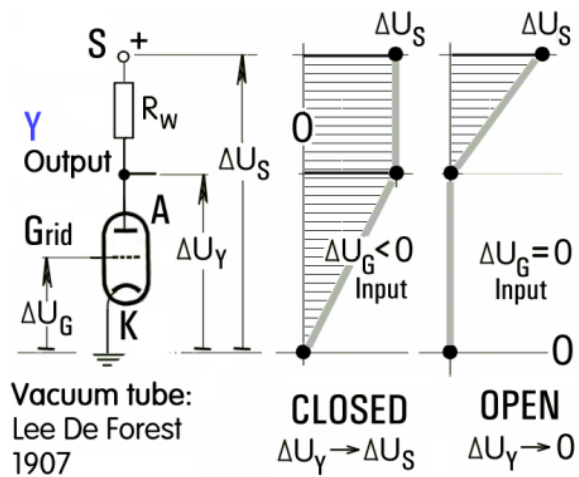

Figure 2. Principle of the current control valve used by Abraham and Bloch in their electronic oscillator. Negative input control voltage $\Delta U_{G}$ applied to grid closes the current of electrons from the cathode $K$ to anode $A$. The consequent absence of voltage drop on the working resistor $R_{w}$ increases the output voltage $\Delta U_{Y}$.

The idea of the anti-parallel circuits appeared in early history of vacuum-tube electronics towards the end of the first world war. The current control in vacuum tubes operates as shown in Fig. 2, by switching between. the two alternative regimes described as OPEN and CLOSED state. The current passing through the vacuum valve is controlled by admission of negative voltage $\Delta U_{G}$ to the grid positioned inside the valve between its cathode $\mathrm{K}$ and anode $A$. If there is no negative voltage applied on the grid, the vacuum is open for motion to the anode $A$ of electrons emitted from the heated cathode $\mathrm{K}$. The motion is driven by voltage difference $\Delta U_{S}$ between $K$ and $S$. The vacuum tube is connected in series with working

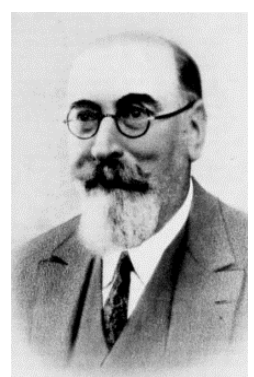

Figure 3. Eugene Bloch (1878-1944), one of the two inventors of the electronic "multivibrateur" - the ancestor of oscillators with two anti-parallel active devices. 

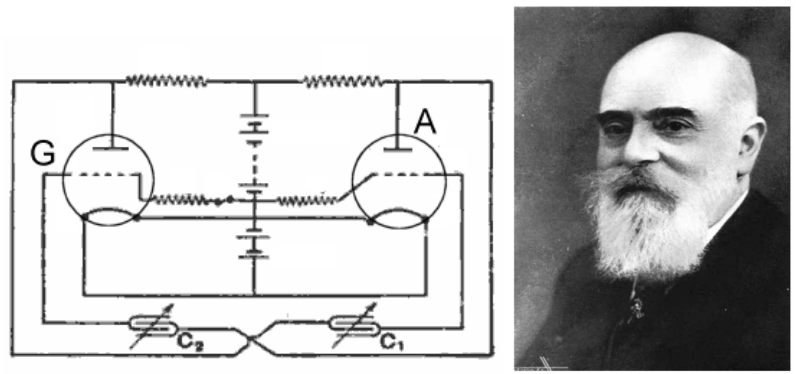

Figure 4 (Left) The original circuit diagram of the "multivibrateur" as it appeared in the 1919 publication ref. [21]. Anode $A$ of one valve is coupled to the grid $G$ of the other valve by means of the capacitors $\mathrm{C}_{1}$ and $\mathrm{C}_{2}$ which cause time delays. Figure 5 (Right) Henri Abraham (1868-1943) - the other one of the two inventors of the idea of anti-parallel valves.

resistor $R_{w}$. The output signal voltage $\Delta U_{Y}$ is taken from the location between this resistor and the valve. As shown in the idealised voltage distributions at the left-hand part of Fig. 2, in the OPEN regime the voltage difference between the output terminal $\mathrm{Y}$ and the cathode $\mathrm{K}$ is practically zero. The whole supplied voltage difference $\Delta \mathrm{U}_{\mathrm{S}}$ is present as voltage drop on the working restrictor $R_{\mathrm{w}}$. The output signal voltage $\Delta U_{\mathrm{Y}}$ is zero.

Once the control voltage $\Delta \mathrm{U}_{\mathrm{G}}$ is applied, the motion of electrons in the resultant CLOSED regime is blocked. Since no current then passes through the working restrictor $R_{w}$, there is practically no voltage trop on it. The whole voltage difference $\Delta U_{S}$ is now available as the output signal between $\mathrm{Y}$ and $\mathrm{K}$.

This description of vacuum tube behaviour helps in understanding what experienced the inventors E. Bloch (Fig. 3) and H. Abraham (Fig. 5), in the study of their interesting circuit with two vacuum tubes in anti-parallel, i.e. one in the OPEN and the other CLOSED regimes and mutually connected, as is apparent from Fig. 4. Note that the anode of one valve is connected to the grid on the other side. This connection is through capacitors $C_{1}$ and $\mathrm{C}_{2}$. These cause a time delay (the time needed for charging the capacitor, so that a particular configuration of currents and voltages has a chance to exits briefly before it is switched off). Condensers, unable to transfer DC current, prevent the circuit from remaining in one of the regimes permanently.

Abraham and Bloch called their circuit a "multivibrateur". Priority of their invention was sometimes considered doubtful because they made the tests of this circuit during the first world war while working for the French Ministère de la Guerre. This, naturally enough during the war, demanded keeping the invention secret. It could be first described in open literature ref. [7] later, after the war in 1919. Prior to this publication, however, two British electrical engineers, Prof. Eccless (Fig. 7) and a technician in his laboratory, W. Jordan (Fig. 8) have applied in 1918 for patent on essentially the same circuit as presented in Fig. 6 (illustration taken from ref. [6]). Their circuit, also with the pair of mutually blocking vacuum tubes, became known as "flip-flop" because, in contrast to the Abraham-Bloch "multivibrateur" the signal transfer was by DC connection. This made the circuit bistable, remaining permanently in one of the two regimes until the arrival of a switching input signal. Nevertheless, the monostable version (with one regime
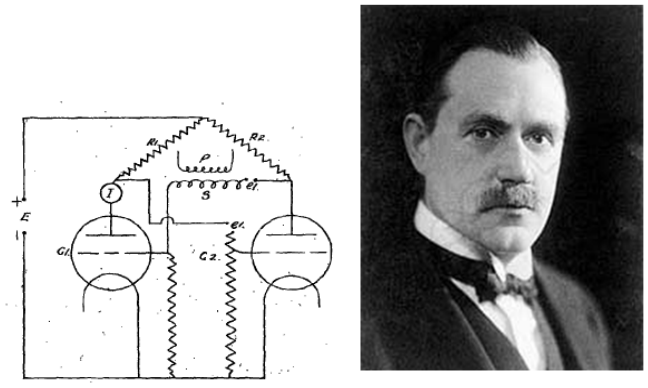

Figure 6 (Left) The original circuit diagram of the "flip-flop" from the 1918 patent. It remains stably in one of two alternative regimes.

Figure 7 (Right) Professor William H. Eccless (1875-1966) who, with his technician Frank Jordan, invented essentially a year before the same circuit as Abraham and Bloch - except that in its standard version the circuit was not set up to oscillate.

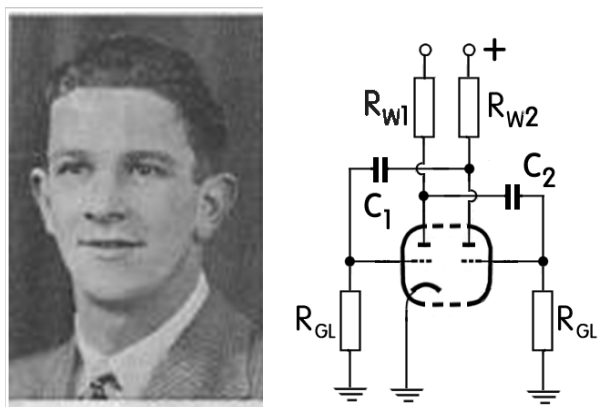

Figure 8 (Left) Frank Wilfred Jordan (1882-?) - the researcher who by toying with electronic circuits in Eccless' laboratory discovered the interesting behaviour of anti-parallel valve connection.

Figure 9 (Right) Eccles-Jordan flip-flop circuit in the oscillator version (note the capacitors $\mathrm{C}_{1}$ and $\mathrm{C}_{2}$ )

preferred) and "astable", i.e. oscillating, were also soon tested. The bistable version was later used in large numbers in the earliest digital computers - in the natural realisation of binary algebra operations. The large amount of these units deserved producing for this purpose special vacuum tubes containing two separate electrode systems in the same glass bulb, as shown in the schematic representation in Fig. 9.

\section{Fluidic bistable turn-down valves}

The electronic circuits, presented schematically in Figs. 6 and 9 , are complicated by added auxiliary components. These, e.g. such as the "grid-leak" resistors $R_{G L}$ in Fig. 9 (they serve for removal of electrons accumulated on the grid), are irrelevant for the adaptation of the basic idea to the fluidic "astable" oscillator. Rather than a closer study of the electronic circuits with these details, it is advisable to follow the basics of the function logic as presented in Figs. 10 and 11, showing the basic logic element and the mutual blockage connection of two such elements $A$ and $B$, respectively. 


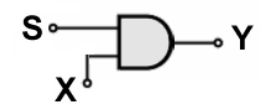

S 1

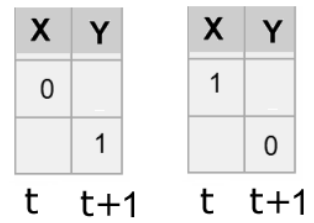

Figure 10. General representation of the basic element performing logical negation after a time step from $t$ to $t+1$. Note the constant supply $S=1$.

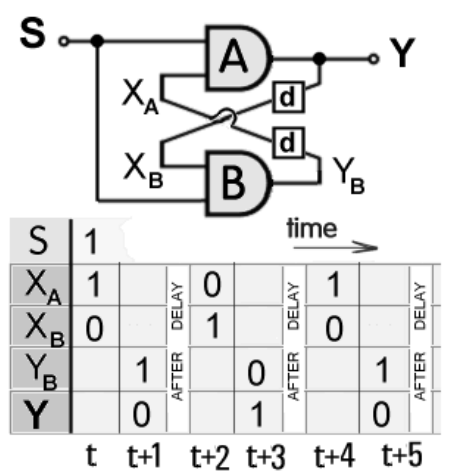

Figure 11. Schematic principle of the Abraham-Bloch oscillating circuit. The output signal in terminal $Y$ switches periodically between the states $Y=0$ and $Y=1$ in each pair of time steps.

The basic problem encountered in an attempt to translate the circuits into the fluidic version is the need of the fluidic valve devices possessing a direct behaviourial analogy to the vacuum tubes. Usual active fluidic devices that might be considered for the role, bistable amplifiers, behave differently. They are not capable of being switched between the OPEN and fully blocked CLOSED regimes. Instead of closing the flow in one their stable regime they divert the fluid flow into another output terminal. If the turning down (not a complete blockage) of a fluid flow is required in no-moving-part fluidics, the solution is to use a vortex amplifier, e.g. [9, 10, 11]. These, however, in their standard version operate not by switching between two regimes. but instead turn the flow down continuously. Although they suffer from two significant limitations:

- Since the basic principle of no-moving-part fluidics is use of inertial forces in fluid (for example the inertia is used to keep a diverted jet flowing into the deflected direction even if it is opposed by the load connected to the outlet), the complete turning down (i.e. closure) of the flow passing through the device is obviously out of question. Stopping the flow would mean an absence of the inertial force. Pure fluidic devices without moved or deformed mechanical components thus can only decrease the flow but cannot stop it. A way out from this dilemma, little known, does exist. It is an addition of a component producing a jet-pump effect. An example is the radial-diffuser jet pump mentioned in [12], with which this limitation is not an unsurmounable one.

- The other until recently serious problem associated with known vortex amplifiers is the fact that their control input pressure must be higher than the pressure in the supply terminal $[13,14]$. This would obviously lead to substantial complication in the design of the anti-parallel circuit, at least if solutions are sought among circuits exhibiting the configuration similarity $\Delta U_{G}<\Delta U_{S}$ of the electronic circuits discussed in Sect. 2.

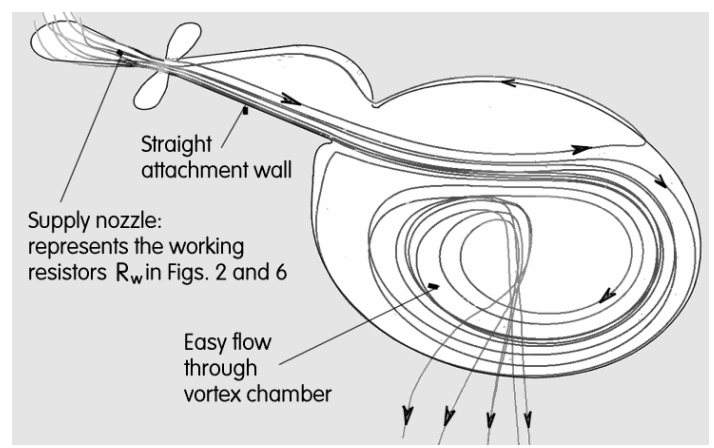

Figure 12. Perspective view of computed $3 D$ pathlines in the author's bistable fluidic device in the OPEN regime of attachment to the straight attachment wall. The pressure drop across the vortex chamber is in this regime very small.

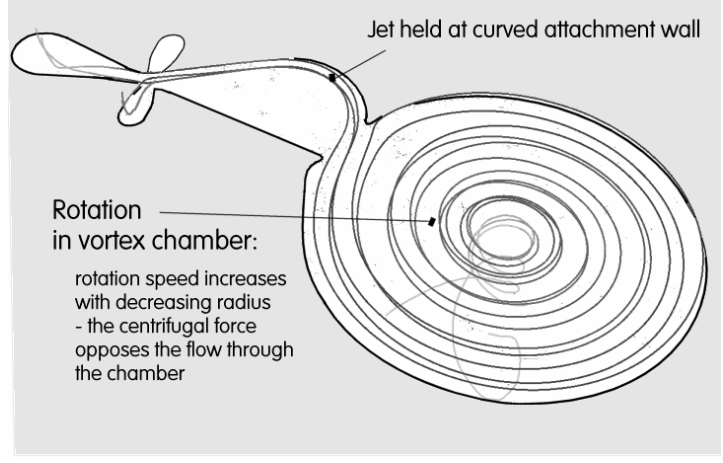

Figure 13. Computed in the same active device as in Fig. 12, is here the fluid flow in the CLOSED regime. The jet from the supply nozzle is attached to the curved attachment wall in the pre-chamber. Large pressure drop across the vortex chamber is due to the necessity of overcoming the centrifugal forces.

Fortunately, a solution to this second, more serious problem has been found in the device discussed in [12] the bistable turn-down valve. From one point of view this valve may be seen as an integrated module consisting of two devices: a bistable diverter upstream and a vortex chamber $[15,16]$ downstream. Another point of view is to see it as a development of the idea of induced rotation [17] in two cavities: here the roughly triangular prechamber and the large round vortex chamber. It is this later point of view that explains the elimination of collectors and diffusers (otherwise unthinkable in a diverter amplifier). Also, this point of view explains the curvature of one of the attachment walls (AW1 in Fig.15) 
and the presence of the single communicating window between the pre-chamber and the vortex chamber.

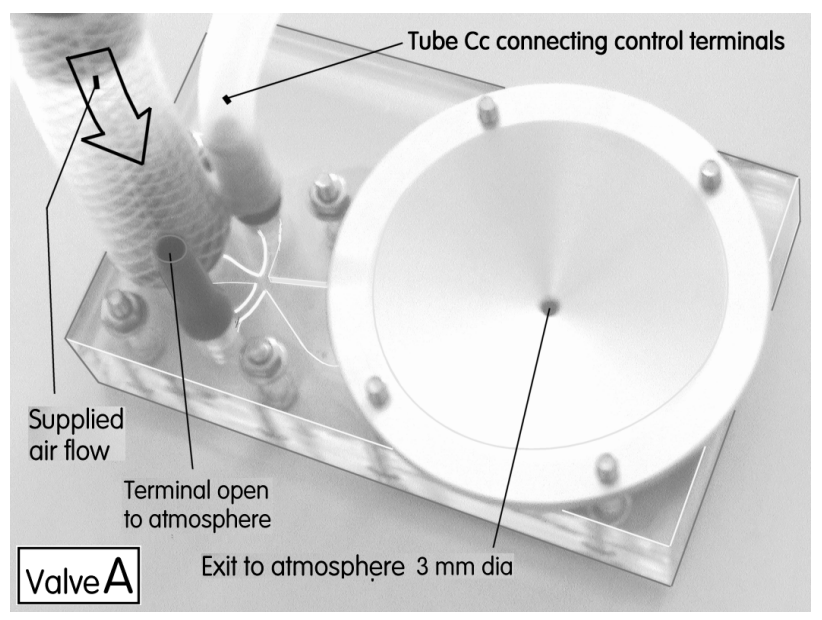

Figure 14. Photograph of the bistable turn-down valve during the performed tests. The terminal $\mathrm{C}_{1}$ is open to atmosphere so that the CLOSED state, with attachment to the curved attachment wall AW1 (figure 15), is possible only under the action of the control inflow into $\mathrm{C}_{2}$

As shown in Fig. 12, in the regime that may be described as OPEN the fluid flow from the supply inlet through this valve manages to get easily into the central exit hole from the vortex chamber lid (the lid is the round aluminium component seen on top of the chamber in the photograph Fig. 14) - with minimal pressure loss. In the other, CLOSED regime - Fig. 13 - the jet issuing form the supply inlet into the pre-camber attaches to the curved attachment wall. This guides it to enter the vortex chamber tangentially. There it begins to rotate.

As the rotation radius decreases, its speed increases (because of conservation - though only approximate - of the fluid moment of momentum). The faster rotation is

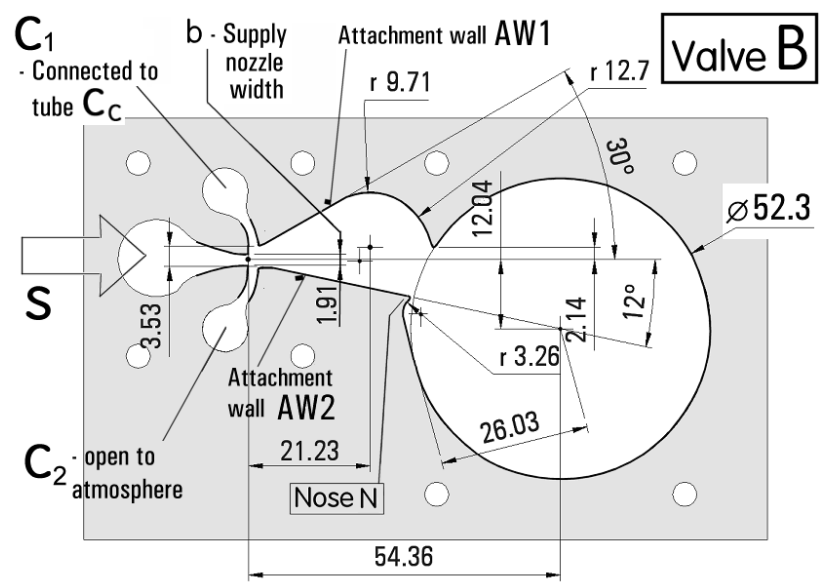

Figure 15. Internal geometry of the two active turn-down bistable valves. Note that $A$ and $B$ valves (Figs. 14, 16, 17) are mutual mirror images.

accompanied with larger centrifugal force acting on the rotating fluid. This force grows to a degree at which it opposes the flow towards the central exit almost completely. Some flow into the output nevertheless remains - it gets to the exit through the less intensively rotating boundary layers on the vortex chamber bottom and lid.

\section{Fluidic circuit with anti-parallel turn- down valves}

To form the anti-parallel fluidic circuit, the two active valves corresponding to Fig. 14 were connected in the manner discussed in the previous Sect. 3. The valves, described as $A$ and $B$ in Fig. 16, were made by laser cutting in a $2 \mathrm{~mm}$ thin PMMA plate, covered from top and bottom by simple flat cover plates. The laser-cut cavities in the two main plates were actually made identical. The required mirror image configuration of Fig. 16 was simply obtained by reversing (top to bottom) one of the main plates.

Both valves were connected to the common supply inlet $\mathrm{S}$, with the exit terminals open into the atmosphere. Also one of their control terminals - in Fig. 16 it is the left one in both valves - was open to atmosphere. The other control terminals are mutually connected by

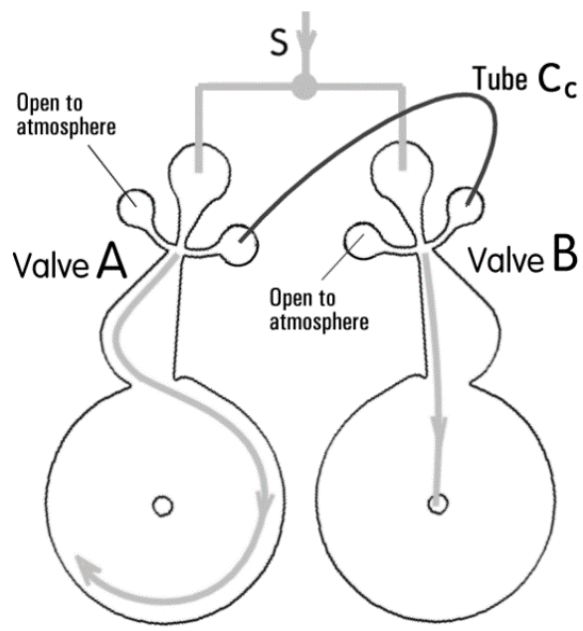

Figure 16. Schematic representation of mutual positions of the two bistable vortex turn-down valves in the tested antiparallel circuit. In contrast to the flip-flops of Sect. 2 there is an immediately apparent asymmetry of the control signal transfer tube $\mathrm{C}_{\mathrm{c}}$.

Both valves are connected to the common supply terminal $\mathbf{S}-$ in the experiments this was an adjustable source of compressed air. The pressure drop across the supply nozzles corresponds to the voltage decrease on the working resistors $R_{w}$ in the electric analogy in Sect. 2. No such fluidic resistors as separate components are therefore necessary. The pressure in the pre-chamber is led through the connecting tube $\mathrm{C}_{\mathrm{c}}$ to the control nozzle of the other valve. The silicone rubber tube $\mathrm{C}_{c}$ is also clearly visible in figure 17 . It was of $620 \mathrm{~mm}$ length and $6 \mathrm{~mm}$ internal diameter. In contrast to the classical "multivibrateur", no such second connection by a tube was in the discussed experiments applied to the other pair of control nozzles, which were both open to the atmosphere (which may be perhaps considered to be also 
a certain form of the interconnection). Of course, the unrestricted inflow of atmospheric air made the corresponding Coanda-effect attachment untenable, so that both devices actually in the present test series were practically monostable. Other variants of control terminal connections were used later in other experimental investigations - the present configuration is remarkable just because of the discovered stochastic regimes.

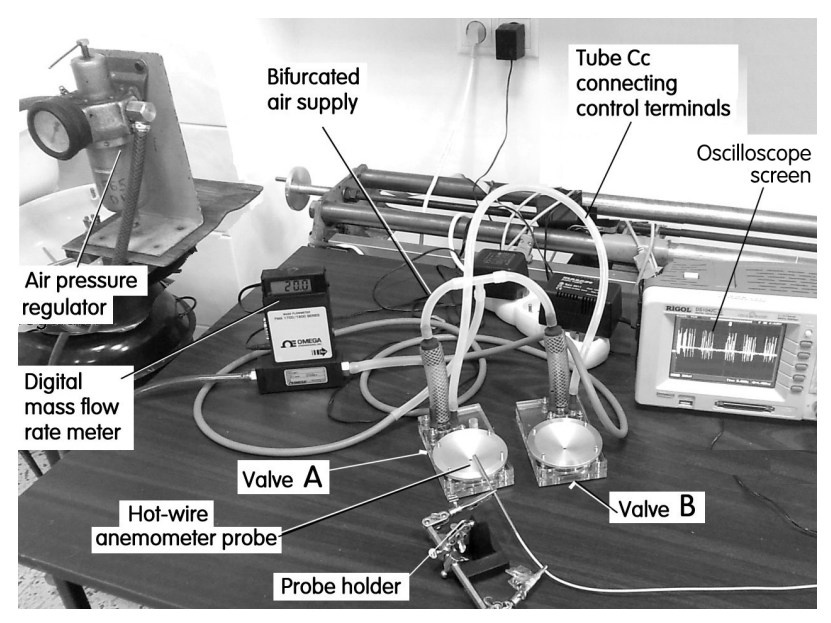

Figure 17. Schematic representation of mutual positions of the two bistable vortex turn-down valves in the anti-parallel circuit in the here discussed experiments.

While the intensity of air flow rotation in the vortex chamber served in the discussed circuit as the variable resistor, increasing or decreasing the fluid flow, the vortex also simultaneously served another purpose. It represented the delay devices (note the necessity of the delays in Fig. 11). The delay was simply caused by the spin-up and spin-down times of the rotating vortex [12, $18]$.

\section{Experiments}

The setup for the tests performed in the laboratory is presented in figure 17. At extreme left in the picture is the pressure regulator used to adjust the compressed air flow. Downstream from it is seen digital mass flow meter FMA 1727A with integral display, capable of measurements in the range 0 to 40 standard litres per minute, calibrated for nitrogen, accuracy $\pm 1.5 \%$ of full scale guaranteed by supplier Omega Engineering Inc. Conversion to values for air, together with $\mathrm{kg} / \mathrm{s}$ units replacing SLM, were made in data processing. The metered air flow then entered the bifurcation into the two supply terminals $\mathrm{S}$.

Availability of only one flowmeter, connected upstream from the bifurcation, caused impossibility of evaluating actual distribution of flow rates between the two devices. After all, the fast changes of the distribution during each period of the investigated oscillation would eould make such measurement impossible anyway, due to the limitations of the flowmeter response (the supplier sytates the settling time of the meter is $800 \mathrm{~ms}$ ). The flow rate was actually used only for indicating the order of magnitude values of Reynolds number and for this purpose it was considered sufficient to work with nominal mean velocity $\bar{w}$ computed under the equipartition condition:

$$
\bar{W}=\frac{M v}{2 b h},
$$

where $\dot{M}[\mathrm{~kg} / \mathrm{s}]$ is the mass flow rate, $\mathrm{v}\left[\mathrm{m}^{3} / \mathrm{kg}\right]$ is specific volume of the gas (air), $b=1.91 \mathrm{~mm}$ is the width of each supply nozzles (cf. figure 15 ), and $h=2$ $\mathrm{mm}$ is the thickness of the plate with the air flow cavities.

The two tested valves $A$ and $B$ are recognisable near the middle of the photograph Fig. 17. The oscillator output was measured by hot-wire MiniCTA anemometer supplied by Dantec. Its probe 55 P 161 was positioned downstream from the exit of the valve $A$. The anemometer signal was displayed on the screen of Rigol oscilloscope, visible at the extreme right of the photograph figure 17 .

\section{Results and discussion}

The onset of oscillation was recognised by audible response at a certain magnitude of the gradually increased supply airflow. There were, in response to further increase, other changes in the character of the air flow. These may be divided into the three regimes presented in the accompanying table, characterised by the values of the nominal Reynolds number:

$$
\overline{R \theta}=\frac{\bar{W} b}{v} .
$$

with $v\left[\mathrm{~m}^{2} / \mathrm{s}\right]$ kinematic viscosity of air.

\begin{tabular}{|l|l|l|}
\hline A & $\begin{array}{l}\text { At small flow rates, } \\
\text { - iust above } \\
\text { oscillation threshold }\end{array}$ & $\begin{array}{l}\text { Pulse packets, } \\
\text { irregulatly appearing } \\
\text { irregular number } \\
\text { of pulses in packet }\end{array}$ \\
\hline (B) & $\begin{array}{l}\text { Medium flow rates } \\
\overline{\operatorname{Re}} \sim 6.510^{3}\end{array}$ & $\begin{array}{l}\text { Individual pulses } \\
\text { a degree of } \\
\text { regularity }\end{array}$ \\
\hline C) & High flow rates & $\begin{array}{l}\text { individual pulses } \\
\text { appearing } \\
\text { at random }\end{array}$ \\
\hline
\end{tabular}

Typical appearances of the oscilloscope screen records in these three regimes are presented in Figs. 18, 19 , and 20.

\subsection{Low $\overline{\mathrm{Re}}$ regime: stochastically appearing pulse packets}

The oscillation signal became audible at roughly $\overline{\mathbf{R e}} \sim$ $\sim 2.510^{3}$ and its character remained more or less the same for increased Reynolds numbers up to $\overline{\mathbf{R e}} \sim 610^{3}$. The example of appearance of the anemometer signal on oscilloscope screen presented in Fig. 18 is inside this range. Dominant feature there are packets of pulses. Typically there were 5 pulses in a packet. Sometimes (especially at lower $\overline{\mathrm{Re}}$ ) the number is increased to 8 and (exceptionally) even more. Sometimes there was the 
other exception, only a single pulse. Frequency of the pulsation in a packet was of the order $\mathrm{f} \sim 20 \mathrm{~Hz}$ (note the value $f=17.3 \mathrm{~Hz}$ in Fig. 18) and remained quite constant for given air flow rate. Repetition rate of the packets was quite stochastic. Evaluation from many recorded cases indicated mean period $0.589 \mathrm{~s}$, corresponding to frequency $1.7 \mathrm{~Hz}$.

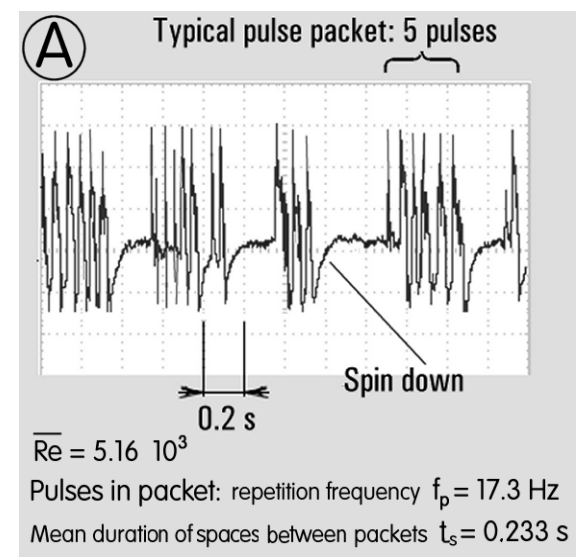

Figure 18. Typical oscilloscope screen record of oscillator output at small flow rates - the regime (A). The oscillation, more or less regular, stops after several pulses to re-appear later. The repetition frequency as well as the no-oscillation space between the pulse packs are quite irregular.

An interesting feature found at the end of each packet is the exponential character of the variation of the output flow velocity signal (labelled "spin-down" in Fig. 18)

The irregularity of pulse generation may be obviously inconvenient in some applications that might be possibly considered for this oscillator, but there is no particular request for regularity in the generation of microbubbles in a bioreactor, which was the raison d'etre of the performed investigation. On the other hand, the capability to attain low oscillation frequencies with repeated sudden clearing bursts may be a distinct advantage - especially when obtained in a very compact oscillator device.

\subsection{Middle $\overline{\mathrm{Re}}$ regime: single flow pulses}

The record presented in the next Fig. 19 shows a phenomenon observed with continuing increase of the supplied air flow rate. It is a distinct decrease of the number of pulses in a packet - finally leading to there being only a single pulse. Another feature there is again the remarkable spin-down, apparent at the end of each pulse. Although there is no exact regularity in the repetition of the pulses, the deviations from the mean period in this regime were quite small.

In the case of the record presented in Fig. 19 the mean period between the generated pulses was $0.325 \mathrm{~s}$, which means the frequency of repetition $f \sim 3 \mathrm{~Hz}$. This is higher than was the frequency of repetition rate of the packets in the low $\overline{\mathbf{R e}}$ regime of Fig. 18, nevertheless still very low indeed.

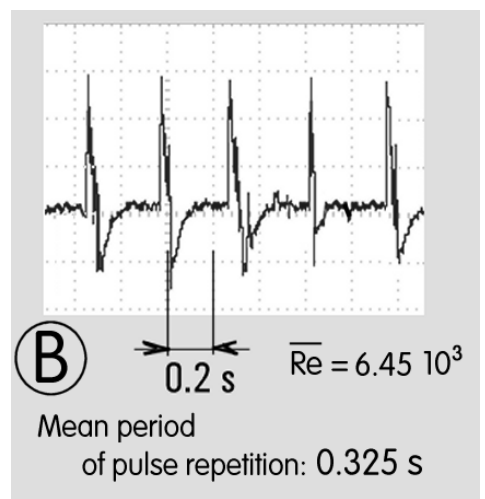

Figure 19. Typical oscillogram recorded in the regime $(\mathbf{B})$. The pulse packets of Fig. 18 are here reduced to single pulses. There is some visible regularity in their repetition.

\subsection{High $\overline{\mathrm{Re}}$ : pulses at very long intervals and exponential dependence}

A result of the experiments that was the least expected one was discovered at the highest supplied air flow rates. The more or less regular repetition rate of Fig. 20 disappeared and was replaced by isolated pulses mutually separated by very long intervals. The example presented in Fig. 20 shows one of these intervals lasting more than one second - but followed by a much shorter one, persisting for only one half of a second.

To evaluate the governing law of the pulse appearances, this obviously stochastic behaviour was investigated applying the classical statistical processing. Time axis was divided into "windows" lasting $0.2 \mathrm{~s}$ and in a very large number of recorded samples was counted the number of pulses in each such "window".

What was expected as a result of the plotting the number of pulse duration occurrences as a function the

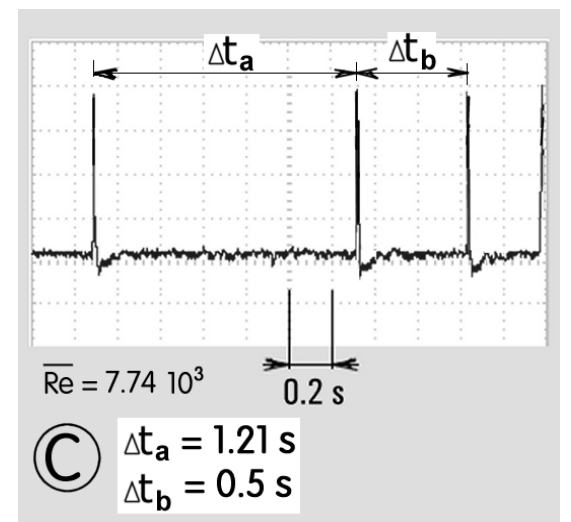

Figure 20. Typical oscillogram of output flow pulsations appearing in the highest $\overline{\mathbf{R e}}$ regime $(\mathbf{C})$. Characteristic are the wide differences in time delays between the pulses. 


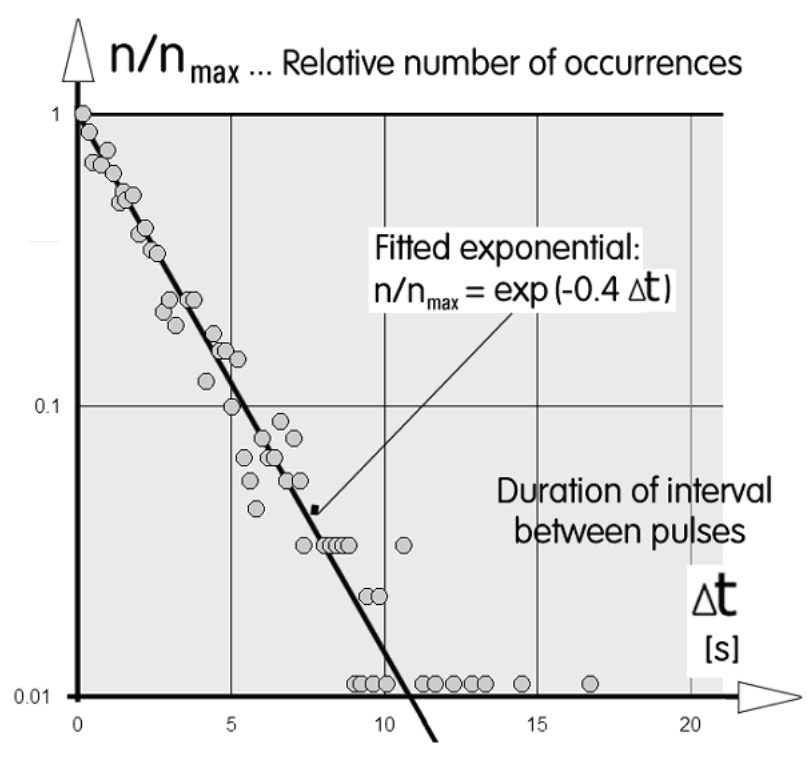

Figure 21. Interesting result of the statistical processing of the lengths (durations) of the interval between the pulses in the regime $(\mathbf{C})$. The number $\mathrm{n}$ of occurrences of the interval lengths inside the 200 ms "windows" was counted in sample ensembles consisting of more than 50 oscilloscope records.

time interval between the pulses was a curve similar to the Gauss' distribution, with a maximum at the most probable interval lengths.

What was actually found is shown plotted in semilogarithmic scale diagram in Fig. 21. The most probable interval between pulses is the shortest one. Number of occurrences then decreases in a monotonic manner - so that were at the end of the diagram also pulses that appeared after more than 10 seconds of waiting for them (the resolution, determined by the $0.2 \mathrm{~s}$ width of the "windows", is seen to be too crude for details of this part of the diagram). The semi-logarithmic scale in Fig. 21 made possible as the nest fitting through the data points the exponential decrease line. The slope of the lines depended on the air mass flow rate. In the particular case of Fig. 21, the fitted line indicates the characteristic time $1 / 0.4=2.5$ seconds.

\section{Conclusions}

In an investigation inspired by the search for microbubble generating, very low frequency fluidic oscillator was tested the idea of a circuit with two controlled flow valves operating in anti-parallel, with mutually blocking interconnection of their control inlets. In the basic principle this corresponds to the idea of Abraham \& Bloch "multibrateur", invented the very beginnings of electronics. The fluidic valves used are of the littleknown bistable amplifiers version with attachment walls pre-chamber and flow turning down vortex chamber.

Three different self-excited oscillation regimes were found, dependent upon the supplied fluid flow rate. The low flow rate regime (A) is characterised by multi-pulse packs with stochastic appearance and duration. More regular behaviour was found in the regime (B), with individual pulses and a degree of periodicity. The most surprising is the regime (C), with pulses seemingly appearing at random - but the interval separation between them, of the order of seconds, revealing extraordinary exponential dependence.

While the idea of a stochastic bubble generator may sound strange, algae do not care for the regularity in the $\mathrm{CO}_{2}$ supply so that the behaviour may be acceptable. There seem to be even some other used of this idea.

\section{Acknowledgements}

Author obtained support from GAČR - Grant Agency of the Czech Republic, by research grant Nr. 13-23046S. There was also institutional support RVO:61388998.

\section{References}

1. Biofuel from algae, eds: A. Padney et al., Elsevier, 2014

2. W. Zimmerman, V. Tesař, British Patent GB20060021561 filed Oct. 2006

3. V. Tesař, Chemical Engineering Journal, 235, 368, (2014)

4. V. Tesař, S. Zhong, R. Fayaz, AIAA Journal, 51397 (2013)

5. V. Tesař, Chemical Engineering Research and Design 921603 (2014)

6. V. Tesař, C.-H. Hung, W. Zimmerman, Sensors and Actuators A: Physical 125159 (2006)

7. Raghu S., Experiments in Fluids 54, 1455 (2013)

8. V. Tesař, Chemical Engineering Science 116 Part I: 843, Part II: 849

9. D. N. Wormley, H. H. Richardson, Journal of Basic Eng. 92369 (1970)

10. R. J. Woolhouse, J. R. Tippetts, S. B. M. Beck, Proc. Inst. Mech. Eng., Part C: J. Mech. Eng. Sci., 215893

11. N. Syred, J. K. Royle,Second IFAC Symposium on Fluidics, Prague, paper B5 (1971)

12. V. Tesař , E. Smyk E., Chemical Engineering and Processing: Process Intensification 90, 6 (2015)

13. D. Parker, M. J. Birch, J. Francis, J. Fluids Eng. 133, 041103 (2011)

14. J. Francis, M. J. Birch, D. Parker, J. Fluids Eng 134, 021103 (2012)

15. V. Tesař V. Messen-steuern-regeln 26, Nr. 4 (1983)

16. V. Tesař V., Proc.gs of the 9-th International Fluidics 'Jablonna' Conf., Jablonna, Poland, Paper A-5, Sept. 1982

17. V. Tesař., EPJ Web of Conferences 92 , article 02096 (2015)

18. V. Tesař, paper \#206, Proc. 21st Internat. Conf. Engineering Mechanics 2015, p. 326, Svratka, Czech Republic, May 11 (2015)

19. V. Tesař, Pressure-Driven Microfluidics, Artech House, Boston, USA (2007)

20. W.H. Eccles, F.W. Jordan, British Patent Nr. 148582, filed June 1918

21. H. Abraham, E. Bloch, Annales de Physique 9237 (1919) 